\title{
PCR-SSCP: A Simple and Sensitive Method for Detection of Mutations in the Genomic DNA
}

\author{
Kenshi Hayashi \\ Oncogene Division, National Cancer Center Research Institute, Tokyo 104, \\ Japan
}

\begin{abstract}
echnical progress in the determination of DNA sequence has resulted in an enormous amount of DNA sequence data, with technical innovations such as the polymerase chain reaction (PCR) accelerating the rate of increase of this data. This combination of data and techniques makes it possible now to exploit sequence differences between individuals. Such studies not only can answer biological questions, but also have potential clinical use in the diagnosis of cancer and hereditary diseases. Making comparative studies between individuals inevitably deals with a large number of samples, thus the methods employed in such studies should be simple.

One such method is PCR-singlestrand conformation polymorphism (SSCP) analysis. Here, the target sequence is first labeled and amplified simultaneously by the PCR of the genomic $\mathrm{DNA}^{(1)}$ or $\mathrm{CDNA}^{(2)}$ using labeled substrates. The PCR product is then denatured and resolved by polyacrylamide gel electrophoresis, and mutations are detected as altered mobility of separated single strands in the autoradiogram. Thus, the overall procedures are rapid and simple. The mutation can be characterized further by eluting the mutated allele from the gel used for autoradiography and amplifying again for sequence determination. ${ }^{(3)}$
\end{abstract}

\section{PRINCIPLE}

Electrophoretic mobility of a particle in a gel is sensitive to both its size and shape. In non-denaturing conditions, single-stranded DNA has a folded structure that is determined by intramolecular interactions, and there- fore, by its sequence. In SSCP analysis, a mutated sequence is detected as a change of mobility in polyacrylamide gel electrophoresis caused by its altered folded structure. Kanazawa et al. ${ }^{(4)}$ described fragments of cloned mutated $\mathrm{F}_{1}$-ATPase gene of Escherichia coli that move anomalously in strand separation gel electrophoresis. ${ }^{(5)}$ It may seem that only limited sequence changes can cause detectable structural change of the molecule. However, it was found that because of its high resolving power, polyacrylamide gel electrophoresis can distinguish most conformational changes caused by subtle sequence differences such as one base substitution in a several-hundred-base fragment. At present, it is not possible to predict the shift of electrophoretic mobility induced by the mutation. Conversely, measurement of the mobility of directionally mutated sequences may provide an empirical approach to the prediction of higherorder structure of single-stranded nucleic acids.

In PCR-SSCP analysis, changes in several hundred bases are detected, in contrast to some other techniques in which changes in relatively short (a few to 20-base) sequences are detected. Thus, PCR-SSCP analysis is much more sensitive to the replication errors that occur during the PCR. However, theoretical calculations based on the recent estimation of error rate of Thermus aquaticus (Taq) DNA polymerase indicate that such errors contribute to at most several percent of the final amplified fragment several hundred bases long. ${ }^{(6)}$ In addition to this, since the errors can be assumed to occur randomly, any particular erroneous se- quence will never become a significant subpopulation if the PCR is started from the usual concentration of genomic DNA (more than $10^{4}$ molecules per reaction). If, however, the reaction is started from a much smaller number (e.g., 10) of molecules, errors in the early cycles of amplification can produce erroneous bands in the PCRSSCP analysis. ${ }^{(7)}$ In such cases, confirmation by independent reaction is necessary.

\section{PRACTICE}

In PCR-SSCP, either labeled primers or deoxynucleotides are used in the PCR. Primers can be efficiently labeled in a batch sufficient for many analyses using crude $\left[\gamma_{-}{ }^{32} \mathrm{P}\right] \mathrm{ATP}$ and polynucleotide kinase. ${ }^{(8)}$ A high concentration of ATP should be used for efficient labeling because the $K_{\mathrm{m}}$ of polynucleotide kinase to ATP is relatively high. Also, the concentration of ATP should be equal to or in excess of the combined concentration of primers to avoid uneven labeling between primers. The labeled primers can be used for the PCR without further purification steps. ${ }^{(9)}$ Labeled deoxynucleotide is added directly to the PCR, thus, the procedures are simpler and more suitable when analyzing a small number of samples (e.g., less than 10). However, labeling with radioactive deoxynucleotides is more expensive. Also, the specific activity of the PCR product is significantly lower than when labeled primers are used, and the radioactivity in the two strands of the PCR product may be different, depending on the base composition of the amplified sequence.

The amount of product required in 
PCR-SSCP for detection is much less than that in some other PCR-based techniques in which products are detected by staining or by other indirect methods. This means that volumes of the reaction and concentrations of substrates can be reduced for lower running cost and less radioactive hazard. We found that the PCR in $5 \mu l$ using primers at $25 \mathrm{nM}$ (when using labeled primers) or deoxynucleotide at $10 \mu \mathrm{M}$ (when using labeled deoxynucleotide) gives satisfactory results for most sequences. ${ }^{(8)}$ Perhaps a small volume is advantageous for strict temperature control, and low concentrations of primers and deoxynucleotides increase the specificity of annealing of primers to templates and reduce the rate of misincorporation by the Taq DNA polymerase. ${ }^{(10)}$ Shorter fragments are better suited for detection of mutations in the SSCP gel. Therefore, usually fragments of less than $400 \mathrm{bp}$ are amplified. Alternatively, longer fragments can be amplified using labeled radioactive deoxynucleotides, and the uniformly labeled PCR products are then digested by restriction enzymes (D. Yandell, pers. comm.). Samples are then denatured and applied to nondenaturing polyacrylamide gel.

The detection of mutations depends on the conformational changes of the single-stranded molecule induced by the mutation, and therefore, sensitive to physical environment in the gel, e.g., temperature, concentration of ions, and solvents. A temperature rise during electrophoresis is especially hazardous for obtaining reproducible results. For this reason, little ohmic heating and efficient cooling are important; also, the use of a thin gel $^{(1)}$ has obvious advantages. Vigorous air cooling and attachment of an aluminum plate on one side of the gel $^{(1)}$ or use of a water-jacketed electrophoretic apparatus (personal observation) is effective for keeping the temperature even throughout the gel and constant during the run. It should be noted that the mobility of singlestranded DNA and the shift caused by mutation can change dramatically if the temperature of electrophoresis is different $\left(4^{\circ} \mathrm{C}\right.$ versus room temperature, which is mostly $20-26^{\circ} \mathrm{C}$; ref. 1 ).

It has been known that complementary single strands are better separated in gels with low crosslinking. ${ }^{(5)}$ The same rule holds true for SSCP gels in detecting mutations. The extent of cross-linking is expressed by $\% \mathrm{C}$, a ratio of the percent concentration of $N, N^{\prime}$-methylenebisacrylamide to the concentration of total acrylamide monomer. ${ }^{(11)}$ At $5 \% \mathrm{C}$, the gel is the most rigid and has minimal pore size at any given total acrylamide concentration. A gel with a lower $\% \mathrm{C}$ is more soft, has remarkably increased pore size, and seems to be more sensitive to conformation. The increased freedom of the acrylamide fibers may explain these effects. In SSCP analysis, gels at 1 to $2 \% \mathrm{C}$ and 5 to $6 \%$ total acrylamide are commonly used. ${ }^{(8)}$

It has been found empirically that the presence of low concentrations $(5-10 \%)$ of glycerol in a gel frequently improve separation of mutated sequences. ${ }^{12}$ The reason for this is unknown. Perhaps, glycerol, because of its weak denaturing action on nucleic acids, partially opens the folded structure of single-stranded nucleic acids so that more surface area of the molecule is exposed, and thus there is more chance for the acrylamide fibers to sense locally confined structural differences caused by mutation. Glycerol, however, reduces mobility, probably because of its viscosity, especially when electrophoresis is run at $4^{\circ} \mathrm{C}$. It is possible that use of other weak denaturing agents can achieve similar merit in separation without the expense of extended electrophoresis. It should be noted, however, that in rare cases mutated sequences show mobility shift only in gels without glycerol.

In summary, by electrophoresis at room temperature using a $5 \%$ gel having $1 \% \mathrm{C}$ and containing $5 \%$ glycerol, the majority of mutations will be detected. Additional mutations may be found by electrophoresis in gel without glycerol at room temperature or at $4^{\circ} \mathrm{C}$. The choice of multiple conditions of electrophoresis depends on the purpose of one's experiments.

\section{SENSITIVITY}

Estimation of the sensitivity of PCRSSCP is meaningful only when the estimation is based on the observation of mutations in various sequence context. For this reason, estimating sensitivity using many mutants in particu- lar sequences, such as the products of saturation mutagenesis, ${ }^{(13)}$ has not been attempted. Rather, I have collected studies in which PCR-SSCP analyses were done on DNA that is known to carry mutations. Here, I define mutations as being detected if one strand of mutated sequence shows mobility different from that of the reference sequence in at least one electrophoretic condition.

The first example is the RAS family genes. ${ }^{(14)}$ Ten point-mutated sequences (four in $\mathrm{c}-\mathrm{Ki}-\mathrm{R} A \mathrm{~S}$ exon 1 , two in N-RAS exon 2 , and one each in $\mathrm{C}-\mathrm{Ki}-\mathrm{R} A \mathrm{~S}$ exon 2, c-Ha-ras exon 1, c-Ha-RAS exon 2, and N-RAS exon 1) were examined after amplification in 100- to 200-bp fragments, and all mutations were detected as significant mobility shifts in at least one of the separated strands.

In the next example, the $O^{6}$. methylguanine alkyltransferase gene (adaA) of Bacillus subtilis, bacteria were mutagenized and mutant clones sensitive to nitrosoguanidine (i.e., phenotype $a d a A^{-}$) were selected. DNA from six clones was examined by PCR-SSCP using sets of primers, each amplifying -350-bp fragments, to cover the gene. One clone turned out to have a large deletion in the gene and amplification products were absent in some of the PCR. Each of the five mutants revealed a significant mobility shift in one of the amplification products. Subsequent sequencing revealed that all mutants had sequence alterations of single-base substitutions or small deletions in the regions where the shifts were observed. (15)

A different approach was tried by Murakami and Hayashi (unpublished) to estimate the sensitivity of PCR-SSCP analysis. Probability of shift of mutated strand $(x)$ can be estimated from the ratio $(R)$ of cases where both strands are shifted $\left(x^{2}\right)$ to cases where only one strand is shifted $[2 x(1-x)]$. This estimation assumes that whether or not one mutated strand shifts is independent of the chance of shift of its complementary strands, and it does not require prior knowledge of whether or not the examined sequences have mutations. Murakami and Hayashi examined genomic and cDNA sequences of the p53 gene and the retinoblastoma susceptibility gene by amplifying various lengths of frag- 
ments from the DNA or RNA of surgically obtained hepatoma tissues. Mutations that induced mobility shift in both strands as well as only in one strand were then counted for the mutations that were found to carry a one-base substitution by subsequent sequencing. From observations of 30 such mutations, it was concluded that using gels with $5-10 \%$ glycerol, $97 \%$ of mutations in 100- to 300-base-long strands caused mobility shift; this value drops to $67 \%$ for strands of 300-450 bases long. These estimations are translated to a sensitivity for PCRSSCP analysis (probability of detecting at least one strand shifted) of more than $99 \%$ and $89 \%$ for 100 - to $300-$-, and 300 - to 450 -bp fragments, respectively. It was also found that the sensitivity is much lower in gels without glycerol.

\section{COMPLICATIONS}

Occasionally, one strand is separated into two or more bands in the SSCP gel, even though the sequence is the same. ${ }^{(1,2)}$ This suggests that the strands having the same sequence can have different stable conformations. Usually, intensity of these conformer bands may be different, but the ratio of the intensity between them is constant from sample to sample. Therefore, such sequence-different conformation situations are easily diagnosed.

In rare cases, one or both strands are detected as smears rather than bands. Conceivable explanations are: (1) The strands are at a transitional state of two (or more) conformations under the electrophoretic condition, and a subtle difference of conditions in the gel, such as a temperature difference between the region near the glass plate and the middle of the gel, is enough to allow different conformations. (2) The strands are at an equilibrium of two (or more) conformations, and flipping to other conformation is too infrequent to be averaged during the electrophoresis. Whatever the reasons, sharp bands are usually obtained by running the electrophoresis under different conditions, such as with or without glycerol.

\section{EXAMPLES}

Development of cancer is a multistep, multipathway event, in which various genetic changes seem to be involved. Various somatic mutations of RAS oncogene families, ${ }^{(14)}$ the p53 gene, ${ }^{(16-19)}$ and the gene responsible for retinoblastoma $^{(2)}$ in cancer cell lines and in surgically obtained cancer tissues have been detected by PCR-SSCP analysis. The search for mutations in clinically obtained cancer cells by conventional methods has been difficult because such specimens are often heavily contaminated with surrounding normal tissues and inflammatory cells. However, by the recently published technique of allele-specific PCR, mutated sequences that constitute only a small portion in the majority of normal sequence can still be characterized unambiguously. ${ }^{(3)}$ Further study of genetic changes in tumors of various cell origin, histological type, and other characters such as drug-resistance may lead to establishment of DNA-based diagnosis of cancer.

Perhaps one obvious use of PCRSSCP is detection of DNA polymorphisms. Several polymorphisms have been detected in the p53 gene locus while searching for mutations in these genes. ${ }^{(16,17)} \mathrm{A}$ polymorphism in the dopamine $D_{2}$ receptor gene was detected by PCR-SSCP, and this polymorphism was used to disprove association of the gene with alcoholism. ${ }^{(20)}$ Polymorphisms in certain repetitive sequences can be detected by PCR-SSCP if the primer sequences for the PCR are chosen in the neighboring single-copy region. Using PCR-SSCP, Orita et al. ${ }^{(21)}$ have detected polymorphisms in about half of examined $A l u$ repeats. Because of their ubiquitous distribution, Alu repeats should be useful as a rich source of polymorphic DNA markers in the construction of a high-resolution linkage map of human genome.

Cystic fibrosis is an autosomal recessive disorder, and 1 in 25 Caucasians are estimated to be carriers of the defect in the cystic fibrosis transmembrane conductance regulator (CFTR) gene responsible for this disorder. Approximately $70 \%$ of the defect was identified to be a deletion at a putative ATP binding domain of this huge $(170 \mathrm{kD})$ protein. Finding other mutations is important, because a much higher percentage of detection is required before the DNA-based diag- nosis of this disorder can be transferred to clinical use. PCR-SSCP was used to detect several new mutations in the CFTR gene. ${ }^{(22,23)}$ Other mutations may be found by a larger-scale survey of DNA from patients and carriers for mutations in various regions of the gene. PCR-SSCP was also successfully used to detect mutations in genes responsible for neurofibromatosis type $1,(24)$ the B1 variant of Tay-Sachs disease, ${ }^{(25)}$ and hemophilia B. ${ }^{(26)}$

Nalidixylic acid is an inhibitor of gyrase. Studies of a few Escherichia coli mutants resistant to the drug revealed amino acid changes clustered near the active center at the amino-terminal region of the A subunit of this enzyme. Munakata et al. (pers. comm.) have isolated 110 nalidixylic acid-resistant mutant clones of $B$. subtilis, and have examined their gyrase A gene by PCRSSCP using a primer pair that amplified a 280-bp fragment which included the corresponding region of the gene (pers. comm.). They found that all nal ${ }^{\mathrm{r}}$ clones gave bands different from that of wild type and that the mutated bands were grouped into three types. Direct sequencing of these mutants $(11,10$, and 3 clones of each type) confirmed that clones which gave the same type of SSCP bands carried the same mutated sequence. Consequently, it was concluded that the A subunit of gyrase is the sole target of nalidixylic acid, and that resistance to this drug can be acquired only by limited mutations near the active center of the enzyme.

\section{CONCLUDING REMARKS}

One advantage of PCR-SSCP analysis over other PCR-based techniques for mutation detection is its simplicity. In PCR-SSCP, mutations are detected by the presence of shifted bands rather than by the absence of signal, as is the case in some other detection methods. Therefore, failure in the PCR does not lead to false-positive results. Most mutations in various sequence contexts seem to be detected, although further accumulation of data may be necessary to estimate accurately the rate of detection of this technique.

PCR-SSCP analysis has potential use in clinical DNA diagnosis. But to transfer this laboratory technique to a clinical test system, use of a nonradioactive 
detection method is important. Ainsworth et al. have shown that the bands of SSCP analysis can be detected by silver staining. ${ }^{(25)}$ We have preliminary results, using fluorescence-labeled primers in the PCR ${ }^{(27)}$ and analyzing the products with an fluorescencebased automated sequencing machine, in which mutations can be detected at a sensitivity equal to that of the radioactivity-based PCR-SSCP analysis.

Since PCR-SSCP analysis is a relatively new technique, only a limited number of published results are available. However, because of the features described above, this technique is expected to be used widely in various fields of basic and applied biology.

\section{ACKNOWLEDGMENTS}

I thank Drs. T. Sekiya, H. Kanazawa, M. Orita, Y. Murakami, Y. Suzuki, M. Iizuka, R. Makino, S. Mashiyama, and other people in our laboratory for help and encouragement. This work was supported in part by Grants-in-Aid from the Ministry of Health and Welfare of Japan for a Comprehensive 10Year Strategy for Cancer Control, grants from the Ministry of Science, Education and Culture of Japan, and from the Special Coordination Fund of the Science Technology Agency of Japan.

\section{REFERENCES}

1. Orita, M., Y. Suzuki, T. Sekiya, and K. Hayashi. 1989. A rapid and sensitive detection of point mutations and genetic polymorphisms using polymerase chain reaction. Genomics 5: 874-879.

2. Murakami, Y., M. Katahira, R. Makino, K. Hayashi, S. Hirohashi, and T. Sekiya. 1991. Inactivation of the retinoblastoma gene in a human lung carcinoma cell line detected by single-strand conformation polymorphism analysis of the polymerase chain reaction product of cDNA. Oncogene 6: 37-42.

3. Suzuki, Y., T. Sekiya, and K. Hayashi. 1990. Allele-specific PCR: A method for amplification and sequence determination of a single component among a mixture of sequence variants. Anal. Biochem. 192: 82-84.

4. Kanazawa, H., T. Noumi, and M. Futai. 1986. Analysis of Escherichia coli mutants of the $\mathrm{H}^{+}$-transporting
ATPase: Determination of altered sites of the structural gene. Methods Enzymol. 126: 595-603.

5. Maxam, A.M. and W. Gilbert. 1980. Sequencing end-labeled DNA with base-specific chemical cleavages. Methods Enzymol. 65: 499-560.

6. Hayashi, K. 1990. Mutations induced during the polymerase chain reaction. Technique 2: 216-217.

7. Krawczac, M., J. Reiss, J. Schmidtke, and U. Roisler. 1989. Polymerase chain reaction: Replication error and reliability of gene diagnosis. Nucleic Acids Res. 17: 2197-2201.

8. Mashiyama, S., T. Sekiya, and K. Hayashi. 1990. Screening of multiple DNA samples for detection of sequence changes. Technique 2: 304-306.

9. Hayashi, K., M. Orita, Y. Suzuki, and T. Sekiya. 1989. Use of labeled primers in polymerase chain reaction (LP-PCR) for a rapid detection of the product. Nucleic Acids Res. 17: 3605.

10. Innis, M.A. and D.H. Gelfand. 1990. Optimization of PCRs. In PCR protocols (eds. M.A. Innis, D.H. Gelfand, J.J. Sninsky, and T.J. Thomas), pp. 3-12. Academic Press, San Diego.

11. Chrambach, A. and R. Rodbard. 1971. Polyacrylamide gel electrophoresis. Science 172: 440-451.

12. Orita, M., H. Iwahana, H. Kanazawa, K. Hayashi, and T. Sekiya. 1989. Detection of polymorphisms of human DNA by gel electrophoresis as single strand conformation polymorphisms. Proc. Natl. Acad. Sci. 86: 2766-2770.

13. Meyers, R.M., L.S. Lerman, and T. Maniatis. 1985. A general method for saturation mutagenesis of cloned DNA fragments. Science 229: 242-247.

14. Suzuki, Y., M. Orita, M. Shiraishi, K. Hayashi, and T. Sekiya. 1990. Detection of ras gene mutations in human lung cancers by single-strand conformation polymorphism analysis of polymerase chain reaction products. Oncogene 5: 1037-1043.

15. Morohoshi, F., K. Hayashi, and N. Munakata, submitted.

16. Murakami, Y., K. Hayashi, and T. Sekiya. 1991. Detection of aberrations of the p53 alleles and the gene transcript in human tumor cell lines by single-stranded conformation polymorphism analysis of polymerase chain reaction products. Cancer Res. (in press).

17. Mashiyama, S., Y. Murakami, T. Yoshimoto, T. Sekiya, and K. Hayashi. 1991. Detection of p53 gene mutations in human brain tumors by single-strand conformation polymorphism analysis of polymerase chain reaction products. Oncogene (in press).

18. Sugimoto, K., H. Toyoshima, R. Sakai, K. Miyagawa, K. Hagiwara, H. Hirai, F. Ishikawa, and F. Takaku. 1991. Mutations of the p53 gene in lymphoid leukemia. Blood (in press).

19. Tamura, G., T. Kihana, K. Nomura, M. Terada, T. Sugimura, and S. Hirohashi. 1991. Detection of frequent p53 mutations in primary gastric cancer by cell sorting and polymerase chain reaction-single strand conformation polymorphism analysis. Cancer Res. (in press).

20. Bolos, A.M., M. Dean, S. Lucas-Derse, M. Ramsberg, G.L. Brown, and D. Goldman. 1990. Population and pedigree studies reveal a lack of association between the dopamine $\mathrm{D}_{2}$ receptor gene and alcoholism. $J$. Am. Med. Assoc. 264: 3156-3160.

21. Orita, M., T. Sekiya, and K. Hayashi. 1990. DNA sequence polymorphisms in Alu repeats. Genomics 8: 271-278.

22. Dean, M., M.B. White, J. Amos, B. Gerrard, C. Stewart, K.-T. Khaw, and M. Leppert. 1990. Multiple mutations in highly conserved residues are found in mildly affected cystic fibrosis patients. Cell 61: 863-870.

23. Iannuzzi, M.C., R.C. Stern, F.S. Collins, C.T. Hon, N. Hidaka, T. Strong, L. Becker, M.L. Drumm, M.B. White, B. Gerrard, and M. Dean. 1991. Two frameshift mutations in the cystic fibrosis gene. Am. J. Hum. Genet. 48: 227-231.

24. Cawthon, R.M., R. Weiss, G. Xu, D. Viskochil, M. Culver, J. Stevens, M. Robertson, D. Dunn, R. Gesteland, P. O'Connell, and R. White. 1990. A major segment of the neurofibromatosis type 1 gene: cDNA sequence, genomic structure and point mutations. Cell 62: 193-201.

25. Ainsworth, P.J., L.C. Surh, and M.B. Coulter-Mackie. 1991. Diagnostic single strand conformational polymorphism, (SSCP): A simplified nonradioisotopic method as applied to a 
Tay-Sachs B1 variant. Nucleic Acids Res. 19: 405-406.

26. Demers, D.B., S.J. Odelberg, and L.M. Fisher. 1990. Identification of a factor IX point mutation using SSCP analysis and direct sequencing. Nucleic Acids Res. 18: 5575.

27. Chehab, F.F. and Y.W. Kan. 1989. Detection of specific DNA sequences by fluorescence amplification: A color complementation assay. Proc. Natl Acad. Sci. 86: 9178-9182. 


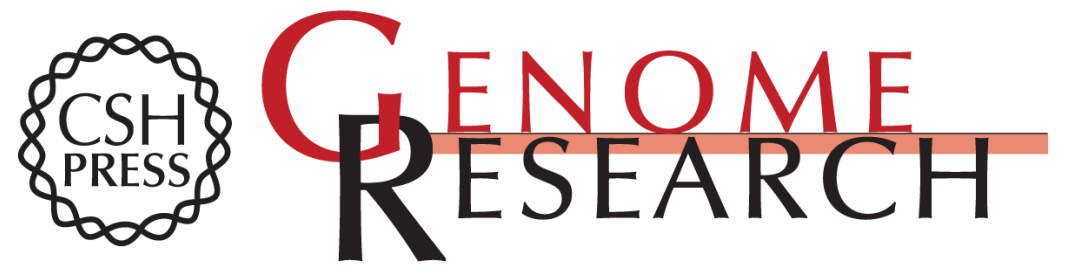

\section{PCR-SSCP: a simple and sensitive method for detection of mutations in the genomic DNA.}

K Hayashi

Genome Res. 1991 1: 34-38

Access the most recent version at doi:10.1101/gr.1.1.34

References This article cites 21 articles, 4 of which can be accessed free at:

http://genome.cshlp.org/content/1/1/34.full.html\#ref-list-1

\section{License}

Email Alerting Receive free email alerts when new articles cite this article - sign up in the box at the Service top right corner of the article or click here.

\section{Affordable, Accurate Sequencing.}

To subscribe to Genome Research go to: https://genome.cshlp.org/subscriptions 